Pacific Journal of Mathematics

ALGEBRAIC ELEMENTS OF A BANACH ALGEBRA MODULO 


\title{
ALGEBRAIC ELEMENTS OF A BANACH ALGEBRA MODULO AN IDEAL
}

\author{
BRUCE A. BARNES
}

\begin{abstract}
Let $A$ be a Banach algebra and $\operatorname{rad}(A)$ its Jacobson radical. It is classical that if $f^{2}-f \in \operatorname{rad}(A)$, then $\exists e \in A$ such that $e^{2}=e$ and $e-f \in \operatorname{rad}(A)$. Calkin and Olsen have proved related results when $A$ is the algebra of all bounded linear operators on a Hilbert space $H$ and the ideal is the ideal of compact operators on $H$. In this paper we consider a Banach algebra $A$ with unit and an ideal $K$ of $A$ and prove generalizations of some of these results.
\end{abstract}

Introduction. Let $A$ be a Banach algebra and let $\operatorname{rad}(A)$ be the Jacobson radical of $A$. A useful classical result in the theory of Banach algebras states that if $f^{2}-f \in \operatorname{rad}(A)$, then $\exists e \in A$ such that $e^{2}=e$ and $e-f \in \operatorname{rad}(A)$ [13, Theorem (2.3.9)]. A related result proved by Calkin in 1941 concerns the Banach algebra $A=\mathscr{B}(H)$, the algebra of all bounded linear operators on a Hilbert space $H$, and the ideal $\mathscr{K}(H)$ of compact operators on $H$. He proved that if $F=F^{*}$ and $F^{2}-F \in \mathscr{K}(H)$, then $\exists E \in \mathscr{B}(H)$ such that $E=E^{2}=E^{*}$ and $F-E \in \mathscr{K}(H)$ [5, Theorem 2.4]. In [12] C. Olsen proved a surprising generalization of Calkin's result: If $p(z)$ is a polynomial and $p(T) \in \mathscr{K}(H)$, then $\exists S \in \mathscr{B}(H)$ such that $p(S)=0$ and $T-S \in \mathscr{K}(H)$. An element $S$ of an algebra is algebraic if $p(S)=0$ for some nonzero polynomial $p$. Thus Olsen's Theorem characterizes an operator that is algebraic modulo $\mathscr{K}(H)$ (a polynomially compact operator) as the sum of an algebraic operator in $\mathscr{B}(H)$ and a compact operator. Other theorems of this type have been proved by $\mathrm{C}$. Akemann and G. Pedersen [1] and by R. Miers [11]. In this paper we look at the general situation of algebraic elements modulo an ideal. The setting is a Banach algebra $A$ and an ideal $K$ of $A$ ( $K$ need not be closed). For convenience we assume that $A$ has a unit (a unit could be adjoined without loss of generality). First we prove a useful lemma concerning when an algebraic element modulo $K$ is the sum of an algebraic element of $A$ and an element of $K$. The result is elementary, yet strong enough to allow us to prove generalizations of many of the results mentioned above and give some other applications besides.

At this point we establish some notation. For $f \in A$ we denote the spectrum of $f$ in $A$ as $\sigma_{A}(f)$ or $\sigma(f)$ when $A$ is understood. If $\sigma$ is a 
spectral set of $f$ (an open and closed subset of $\sigma(f)$ ), then $e(\sigma)$ is the usual spectral idempotent associated with $\sigma[4$, pp. 36-37]. Some of the applications concern algebras of operators. Throughout, $X$ will denote a Banach space, $\mathscr{B}(X)$, the algebra of all bounded linear operators on $X, \mathscr{K}(X)$, the ideal of compact operators on $X, \mathscr{T}(X)$, the ideal in $\mathscr{B}(X)$ of finite rank operators, and $\mathscr{I}(X)$, the ideal of inessential operators on $X$ [6, p. 33]. On the algebraic side, the socle of $A$, denoted $\operatorname{soc}(A)$, plays a role; see [13, pp. 46-47]. In particular we prove an Olsen-type theorem for elements algebraic modulo $\operatorname{soc}(A)$. Note that when $A=\mathscr{B}(X), \operatorname{soc}(A)=\mathscr{T}(X)$.

1. Algebraic elements modulo an ideal. Throughout this paper $A$ is a Banach algebra with unit 1 , and $K$ is an ideal of $A$. Our first order of business is to prove our main tool. This tool reduces many questions concerning algebraic elements in $A$ modulo $K$ to the verification of the following hypothesis, labeled $H_{N}$.

$H_{N}: N$ is a fixed positive integer such that for any $k$, $1 \leq k \leq N$, and any idempotent $e \in A$, if $f \in A$, efe $=f$, and $f^{k} \in K$, then $\exists g \in A, g=e g e, g^{k}=0$, and $\exists h \in K$ such that $f=g+h$.

Note that $H_{1}$ is always true. The tool we use is the following.

Lemma 1. Assume $K$ is an ideal of $A$ which satisfies $H_{N}$. Let $p(z)=$ $\Pi_{j=1}^{m}\left(z-\lambda_{j}\right)^{n_{j}}$ be a polynomial with $1 \leq n_{j} \leq N$ for $1 \leq j \leq m$. If $f \in A$ has the properties

(1) $p(f) \in K$; and

(2) $\sigma(f)=\bigcup_{j=1}^{m} \sigma_{j}$ where $\left\{\sigma_{1}, \ldots, \sigma_{m}\right\}$ is a disjoint collection of spectral sets of $f$ with $\lambda_{j} \in \sigma_{j}$ for $1 \leq j \leq m$; then $f=g+h$ where $p(g)=0$ and $h \in K$.

Proof. For each $j$ let $e_{j}=e\left(\sigma_{j}\right)$. Then $\left\{e_{1}, \ldots, e_{m}\right\}$ is an orthogonal set of idempotents with $1=\sum_{j=1}^{m} e_{j}$. Let

$$
q_{j}(z)=\prod_{k=1, k \neq j}^{m}\left(z-\lambda_{k}\right)^{n_{k}} .
$$

Set $A_{j}=e_{j} A e_{j}$, and note that from spectral theory we have $\sigma_{A_{j}}\left(f e_{j}\right)=\sigma_{j}$. Thus for each $j \exists r_{j} \in A_{j}$ such that

$$
q_{j}\left(f e_{j}\right) r_{j}=e_{j} \text {. }
$$

It follows that

$$
\left(f e_{j}-\lambda_{j} e_{j}\right)^{n_{j}} \in K
$$


By hypothesis $H_{N} \exists g_{j}, h_{j}$ such that $e_{j} g_{j} e_{j}=g_{j}, g_{j}^{n_{j}}=0, h_{J} \in K$, and

$$
f e_{j}-\lambda_{j} e_{j}=g_{j}+h_{j} \text {. }
$$

Let $g=\sum_{j=1}^{m}\left(\lambda_{j} e_{j}+g_{j}\right)$, and $h=\sum_{j=1}^{m} h_{j}$. Note that $f=g+h$ and $h \in K$. It remains to verify that $p(g)=0$. Now for each $j,\left(g-\lambda_{j}\right) e_{j}=g_{j}$, so that

$$
\left(g-\lambda_{j}\right)^{n_{j}} e_{j}=g_{j}^{n_{j}}=0 .
$$

Therefore

$$
p(g)=\left(\prod_{j=1}^{m}\left(g-\lambda_{j}\right)^{n_{j}}\right)\left(e_{1}+e_{2}+\cdots+e_{m}\right)=0 .
$$

Remark. Assume that $\sigma(f)$ is totally disconnected and $\left\{\lambda_{1}, \ldots, \lambda_{m}\right\}$ $\subset \sigma(f)$. Then it is easy to see that there exists a disjoint collection of spectral sets of $f,\left\{\sigma_{1}, \ldots, \sigma_{m}\right\}$ with $\sigma(f)=\bigcup_{j=1}^{m} \sigma_{j}$ and $\lambda_{j} \in \sigma_{j}$ for $1 \leq j$ $\leq m$. Thus in this case (2) of Lemma 1 holds automatically for $f$. This remark applies in the following situation. Let $A=\mathscr{B}(X)$ and $K \subset \mathscr{I}(X)$. If $p(z)$ is a nonzero polynomial such that $p(T) \in K$, then by the Spectral Mapping Theorem $p(\sigma(T))=\sigma(p(T))$ which is a countable set. Therefore $\sigma(T)$ is countable, hence totally disconnected.

For $f \in A$, let $\mathfrak{A}(f)$ be the closed subalgebra of $A$ generated by $f$ and the unit element, and let $(f)^{c c}$ denote the collection of all elements of $A$ that commute with everything that commutes with $f$.

For $\tau$ a compact subset of $\mathbf{C}$, let $\hat{\tau}$ denote the polynomial convex hull of $\tau$ [4, Def. 10, p. 101].

The case where $p(z)$ has simple zeros is of special importance. We derive a corollary concerning this case.

Corollary 2. Assume that the polynomial p has only simple zeros, and assume that (1) and (2) hold in Lemma 1.

(1) The algebraic element $g$ in Theorem 1 can be chosen to be

$$
g=\sum_{k=1}^{m} \lambda_{k} e\left(\sigma_{k}\right) \in(f)^{c c} .
$$

(2) If $\left\{\hat{\sigma}_{, \ldots,} \hat{\sigma}_{m}\right\}$ is a disjoint collection, then

$$
g=\sum_{k=1}^{m} \lambda_{k} e\left(\hat{\sigma}_{k}\right) \in \mathfrak{A}(f) .
$$

Proof. Of course $H_{1}$ is always satisfied by any ideal $K$. Then as constructed in Theorem $1, g=\sum_{k=1}^{m} \lambda_{k} e\left(\sigma_{k}\right)$, and $g \in(f)^{c c}$ since $e\left(\sigma_{k}\right) \in$ $(f)^{c c}$ for all $k$. Thus $(1)$ holds. Now assume that $p(z)$ has simple zeroes 
and $\sigma(f)=\bigcup_{k=1}^{m} \sigma_{k}$ as in (2) of Lemma 1. Suppose further that $\left\{\hat{\sigma}_{1}, \ldots, \hat{\sigma}_{m}\right\}$ is a disjoint collection. By [4, Theorem 11, p. 101]

$$
\sigma_{\mathscr{Y}(f)}(f)=\left(\sigma_{A}(f)\right)^{\wedge}=\bigcup_{k=1}^{m} \hat{\sigma}_{k} .
$$

Therefore

$$
g=\sum_{k=1}^{m} \lambda_{k} e\left(\hat{\sigma}_{k}\right)
$$

is in $\mathfrak{A}(f)$ and has the properties $p(g)=0$ and $f-g \in K$.

If $A=\mathscr{B}(H)$ and $K$ is any ideal, $K \subset \mathscr{K}(H)$, then Corollary 2 applies. However, Olsen has noted that there exists an operator $T \in \mathscr{B}(H)$ such that $T^{2}$ is trace class, but $(T-J)^{2} \neq 0$ for any trace class operator $J$ [12, Example 4.6]. Thus although Corollary 2 holds for $K$, there is no general extension of Corollary 2 to the situation where $p(z)$ does not have only simple zeroes.

Now we give two examples to show that even when solutions $g$ to $p(f-g)=0$ exist, there need not exist a solution that commutes with $f$; and even when there are commuting solutions, there need not be a solution in $\mathfrak{A}(f)$.

EXAMPLE 3. Let $H$ be a separable Hilbert space, and $I$ the identity operator on $H$. Fix an orthonormal basis of $H$, and let $J$ be the diagonal operator relative to this basis with diagonal entries $J_{k k}=1 / k, k \geq 1$. Of course $J \in \mathscr{K}(H)$ and

(1) If $S \in \mathscr{B}(H)$ and $S J=J S$, then $S$ is a diagonal operator.

Now define $T \in \mathscr{B}(H \oplus H)$ by

$$
T=\left(\begin{array}{ll}
0 & J \\
I & 0
\end{array}\right)
$$

Then

$$
T^{2}=\left(\begin{array}{ll}
J & 0 \\
0 & J
\end{array}\right) \in \mathscr{K}(H \oplus H) .
$$

By Olsen's Theorem [12, Theorem 2.4] $\exists R \in \mathscr{K}(H \oplus H)$ such that $(T-R)^{2}=0$. We prove that $R$ cannot commute with $T$. Assume on the contrary that $R T=T R$. Write

$$
R=\left(\begin{array}{ll}
R_{1} & R_{2} \\
R_{3} & R_{4}
\end{array}\right)
$$


From the fact that $T R=R T$, one easily computes that

$$
R_{1}=R_{4}, \quad R_{1} J=J R_{1}, \quad \text { and } \quad R_{2}=J R_{3}=R_{3} J .
$$

Then using (2) and the assumption $(T-R)^{2}=0$ one can derive

$$
R_{1}\left(I-R_{3}\right)=0 \text { and }\left(I-R_{3}\right)^{2} J=-R_{1}^{2} .
$$

By (1) and (2) $R_{1}$ and $R_{3}$ are diagonal operators in $\mathscr{K}(H)$, and thus

$$
\left(R_{1}\right)_{k k} \rightarrow 0 \text { and }\left(R_{3}\right)_{k k} \rightarrow 0
$$

as $k \rightarrow \infty$. Using the equation $R_{1}\left(I-R_{3}\right)=0$, we have that $\left(R_{1}\right)_{k k}=0$ for all but a finite number of $k$. But this contradicts the equation in (3): $\left(I-R_{3}\right)^{2} J=-R_{1}^{2}$. Thus no commuting compact solution of $(T-R)^{2}$ $=0$ exists.

EXAmPle 4. Let $\Omega$ be the compact subset of $\mathbf{C}$ given by

$$
\Omega=\{z \in \mathbf{C}:|z|=1\} \cup\{-2,2\} .
$$

Let $A$ be the algebra of all complex-valued continuous functions on $\Omega$. Let

$$
K=\{g \in A: g(-2)=g(2)=0\} .
$$

Set $f(z)=z$ for $|z|=1, f(-2)=0, f(2)=1$. Then $f^{2}-f \in K$. If $e$ is the idempotent $e(-2)=0$ and $e(z)=1, z \in \Omega \backslash\{-2\}$, then $(f-e) \in K$. But

$$
\sigma_{\mathfrak{A}(f)}(f)=\left(\sigma_{A}(f)\right)^{\wedge}=\{z:|z| \leq 1\} .
$$

Since this set is connected, $\mathfrak{A}(f)$ contains only the idempotents 0 and 1 .

Now we consider several applications. In considering general roots and logarithms in a Banach algebra, the roots and logarithms of the unit element play a significant role; see [9] or [4, p. 43, pp. 89-90]. We look briefly at the situation concerning roots and logarithms modulo an ideal $K$ of operators on a Banach space $X$ where $K \subset \mathscr{I}(X)$. In particular the results apply when $K$ is one of the Von Neumann-Schatten $p$-classes.

We adopt the terminology in [4, p. 89].

Proposition 5. Let $A=\mathscr{B}(X)$ and $K$ be an ideal of $A$ with $K \subset \mathscr{I}(X)$. Assume that $T \in A$ is invertible $\bmod (K)$, and that $\sigma(T)$ is irrotational $\bmod (2 \pi / n)$. If $T^{n}=R^{n} \bmod (K)$, then $T$ and $R$ commute $\bmod (K)$ and $T=S R+J$, where $S$ commutes with $T$ and $R \bmod (K), S^{n}=I$, and $J \in K$.

Proof. That $T R=R T \bmod (K)$ follows as in the proof of $[4, \S 18$, Prop. 11 part (i)] (in the proof interpret equality as equality $\bmod (K)$ ). 
Now let $R^{\prime}$ be an inverse for $R \bmod (K)$ and set $Q=T R^{\prime}$. Then $Q^{n}=I \bmod (K)$ (note that $\left.T R^{\prime}=R^{\prime} T \bmod (K)\right)$. By Corollary $2 \exists S \in$ $\mathfrak{U}\left(T R^{\prime}\right)$ such that $S^{n}=I$ and $T R^{\prime}-S \in K$. Since $T$ and $R$ commute with $T R^{\prime} \bmod (K)$, both operators commute with $S \bmod (K)$.

A result similar to Proposition 5 holds for logarithms modulo an ideal $K \subset \mathscr{I}(X)$. If $T \in \mathscr{B}(X)$ and $f(z)$ is a nontrivial holomorphic function defined on some connected open set $U$ with $\sigma(T) \subset U$, then $f$ has at most a finite number of zeros on $\sigma(T)$. Thus $f$ is of the form $f(z)=p(z) g(z)$, where $g$ is holomorphic in $U$ and has no zeroes on $\sigma(T)$, and $p$ is a polynomial. Therefore if $f(T) \in K$, then $p(T) \in K$ and we can use Lemma 1. These remarks apply to the entire function $f(z)=\exp (z)-1$. These ideas can be used to establish the following result. We omit the proof (see [4, p. 90]).

Proposition 6. Let $A=\mathscr{B}(X)$ and $K$ be an ideal of $A$ with $K \subset \mathscr{I}(X)$. Assume that $T \in A$, and $\sigma(T)$ is incongruent $\bmod (2 \pi i)$. If $\exp (T)=$ $\exp (R) \bmod (K)$, then $T$ and $R$ commute $\bmod (K)$ and $T=(S+R)+J$ where $S$ commutes with $T$ and $R \bmod (K), \exp (S)=I$, and $J \in K$.

Next we consider the classical case where the ideal $K$ is $\operatorname{rad}(A)$. We denote the center of $A$ by $Z(A)$.

\section{THEOREM 7.}

(1) If $p(z)$ has simple zeroes and $p(f) \in \operatorname{rad}(A)$, then $\exists g \in \mathfrak{A}(f)$ such that $f-g \in \operatorname{rad}(A)$ and $p(g)=0$.

(2) If $f \in Z(A)$ and $p$ is a nonzero polynomial with $p(f) \in \operatorname{rad}(A)$, then the minimal polynomial for $f$ modulo $\operatorname{rad}(A)$ has simple zeroes. Thus (1) applies.

Proof. Assume that $p(z)$ has simple zeros and $p(f) \in \operatorname{rad}(A)$. Then $p(\sigma(f))=\sigma(p(f))=\{0\}[13$, Theorem (2.3.2) (iv)], so that $\sigma(f)$ is finite. Therefore (2) of Lemma 1 is automatically satisfied, and Corollary 2 implies that (1) holds.

Now suppose $f \in Z(A)$ and $p(f) \in \operatorname{rad}(A)$ where $p(z)$ is a nonzero polynomial. Since $f \in Z(A)$, we have $e_{k}=e\left(\sigma_{k}\right) \in Z(A)$ for all $k$. As in the proof of Lemma 1,

$$
\left(\left(f-\lambda_{k}\right) e_{k}\right)^{n_{k}} \in \operatorname{rad}(A) .
$$

Now if $h \in Z(A)$ and $h^{n} \in \operatorname{rad}(A)$, then $(A h)^{n} \in \operatorname{rad}(A)$. This implies by [13, Theorem (2.2.9)] that $A h$ is included in every primitive ideal. Since 
$\operatorname{rad}(A)$ is the intersection of all primitive ideals of $A[13$, Theorem (2.3.2)(i)], $h \in A h \subset \operatorname{rad}(A)$. It follows from this argument that

$$
\left(f-\lambda_{k}\right) e_{k} \in \operatorname{rad}(A), \quad 1 \leq k \leq m \text {. }
$$

Let

$$
g=\sum_{k=1}^{m} \lambda_{k} e_{k} \text { and } q(z)=\prod_{k=1}^{m}\left(z-\lambda_{k}\right)
$$

Then

$$
f-g=\sum_{k=1}^{m}\left(f-\lambda_{k}\right) e_{k} \in \operatorname{rad}(A), \quad q(g)=0, \quad \text { and } \quad q(f) \in \operatorname{rad}(A) \text {. }
$$

2. Algebraic elements modulo the socle. In this section we prove that if $A$ is a semisimple Banach algebra, $p$ a polynomial with $p(T) \in$ $\operatorname{soc}(A)$, then $\exists J \in \operatorname{soc}(A)$ such that $p(T-J)=0$. As one application of this result, we have an Olsen-type theorem for $A=\mathscr{B}(X)$ relative to the ideal $K=\mathscr{T}(X)=\operatorname{soc}(\mathscr{B}(X))$.

Let $X$ be a Banach space, and let $X^{\prime}$ be the dual space of $X$. For $x \in X$ and $\alpha \in X^{\prime}$, we write $\alpha(x)=\langle x, \alpha\rangle$. A subspace $\Gamma$ of $X^{\prime}$ is total if $\langle x, \alpha\rangle=0$ for all $\alpha \in \Gamma \Rightarrow x=0$. Also, we use the notation $\alpha \otimes x$ to denote the operator

$$
(\alpha \otimes x)(y)=\alpha(y) x, \quad y \in X .
$$

For $T \in \mathscr{B}(X)$ we denote by $T^{\prime}$ the usual adjoint operator of $T$ on $X^{\prime}$. We let $\mathcal{N}(T)$ denote the null space of $T$.

LEMMA 8. Assume $X$ is a normed linear space and $\Gamma$ is a total subspace of $X^{\prime}$. Let $T \in \mathscr{B}(X)$ be such that $T^{\prime}(\Gamma) \subset \Gamma$. Suppose $\left\{x_{1}, \ldots, x_{n}\right\}$ is a linearly independent subset of $X$, and set $M=\operatorname{span}\left\{x_{1}, \ldots, x_{n}\right\}$. Assume further that $M \cap \mathscr{N}(T)=\{0\}$. Then $\exists\left\{\alpha_{1}, \ldots, \alpha_{n}\right\} \subset \Gamma$ such that $\alpha_{k}\left(x_{J}\right)$ $=\delta_{k j}$ and $\alpha_{k}(\mathcal{N}(T))=\{0\}$.

Proof. Assume $n=1$. If $\left\langle x_{1}, T^{\prime} \alpha\right\rangle=0$ for all $\alpha \in \Gamma$, then $\left\langle T x_{1}, \alpha\right\rangle=$ 0 for all $\alpha \in \Gamma$, and this implies that $T x_{1}=0$, a contradiction. Thus $\exists \alpha \in \Gamma$ with $\left\langle T x_{1}, \alpha\right\rangle=\left\langle x, T^{\prime} \alpha\right\rangle=1$. Set $\alpha_{1}=T^{\prime} \alpha$, and note that $\alpha_{1}(\mathscr{N}(T))=\{0\}$. Now assume that the result holds for $n-1$, and let $\left\{x_{1}, \ldots, x_{n}\right\}$ be as in the statement of the lemma. Choose $\left\{\alpha_{1}, \ldots, \alpha_{n-1}\right\}$ $\subset \Gamma$ such that $\left\langle x_{k}, \alpha_{j}\right\rangle=\delta_{k j}, 1 \leq k \leq n-1$, and $\alpha_{k}(\mathscr{N}(T))=\{0\}$. Define $S$ and $G$ on $X$ as follows:

$$
G=\sum_{k=1}^{n-1} \alpha_{k} \otimes x_{k}, \quad \text { and } \quad S=T(I-G)
$$


Then

$$
\begin{gathered}
\mathscr{N}(T)+\operatorname{span}\left\{x_{1}, \ldots, x_{n-1}\right\} \subset \mathscr{N}(S), \text { and } \\
S x_{n}=T\left(x_{n}-\sum_{k=1}^{n-1} \alpha_{k}\left(x_{n}\right) x_{k}\right) \neq 0 .
\end{gathered}
$$

As in the argument at the beginning of the proof, we can choose $\alpha_{n} \in \Gamma$ such that $\alpha_{n}\left(x_{n}\right)=1$ and $\alpha_{n}(\mathscr{N}(S))=\{0\}$. Then $\alpha_{n}\left(x_{j}\right)=0$ for $1 \leq j \leq$ $n-1$ and $\alpha_{n}(\mathscr{N}(T))=\{0\}$. Thus the result follows by induction.

Now fix $\Gamma$ a total closed subspace of $X^{\prime}$. Let

$$
\begin{gathered}
\mathscr{T}(X, \Gamma)=\operatorname{span}\{\alpha \otimes x: \alpha \in \Gamma, x \in X\}, \quad \text { and } \\
\mathscr{A}(X, \Gamma)=\left\{T \in \mathscr{B}(X): T^{\prime}(\Gamma) \subset \Gamma\right\} .
\end{gathered}
$$

Then $\mathscr{T}(X, \Gamma)$ is an ideal in the algebra $\mathscr{A}(X, \Gamma)$. The previous lemma can be restated as follows:

Suppose $T \in \mathscr{A}(X, \Gamma)$ and $M$ is a finite dimensional subspace of $X$ with $M \cap \mathscr{N}(T)=\{0\}$. Then there exists a projection $F \in \mathscr{T}(X, \Gamma)$ such that $F(X)=M$ and $\mathscr{N}(T) \subset \mathscr{N}(F)$.

Here $F$ is the projection,

$$
F=\sum_{k=1}^{n} \alpha_{k} \otimes x_{k}
$$

where $\left\{x_{k}\right\}$ and $\left\{\alpha_{k}\right\}$ are as in Lemma 8 .

Lemma 9. Assume $T \in \mathscr{A}(X, \Gamma)$ and $T^{n} \in \mathscr{T}(X, \Gamma)$. Then there exists $G \in \mathscr{T}(X, \Gamma)$ such that $[T(I-G)]^{n}=0$.

Proof. Suppose $n=1$. Then since $T \in \mathscr{T}(X, \Gamma)$ we have $X=M \oplus$ $\mathscr{N}(T)$ for some finite dimensional subspace $M \subset X$. By Lemma $8 \exists F$ a projection in $\mathscr{T}(X, \Gamma)$ such that $F(X)=M$ and $F(\mathscr{N}(T))=\{0\}$. Thus $T(I-F)=0$.

Now assume that the result holds for $n-1$, and $T^{n} \in \mathscr{T}(X, \Gamma)$. Set $R=\overline{T(X)}$. Since $T^{n-1} \mid R$ is an operator with finite dimensional range, there exists a finite dimensional subspace $M \subset R$ such that

$$
R=M \oplus\left[R \cap \mathcal{N}\left(T^{n-1}\right)\right]
$$

By Lemma $8 \exists E \in \mathscr{T}(X, \Gamma)$ such that $E(X)=M$ and $E\left(\mathscr{N}\left(T^{n-1}\right)\right)=$ $\{0\}$. Then

$$
(T(I-E))^{n-1}\left(\mathscr{N}\left(T^{n-1}\right)\right)=\{0\},
$$

and it follows that

$$
(T(I-E))^{n-1} \in \mathscr{T}(R, \Gamma)
$$


Applying the induction hypothesis, $\exists F \in \mathscr{T}(R, \Gamma)$ such that

$$
[T(I-E)(I-F)]^{n-1}(R)=\{0\} .
$$

Of course we can consider $F$ as an operator in $\mathscr{T}(X, \Gamma)$. Then

$$
[T(I-E)(I-F)]^{n}(X) \subset[T(I-E)(I-F)]^{n-1}(R)=\{0\} .
$$

This proves the lemma.

Let $A$ be a primitive Banach algebra. By [13, Theorem (2.4.12)] there exist a Banach space $X$ and a total closed subspace $\Gamma$ of $X^{\prime}$ such that $A$ can be represented as a subalgebra of $\mathscr{A}(X, \Gamma)$ and $\operatorname{soc}(A)$ as $\mathscr{T}(X, \Gamma)$. Using this representation, we prove that Lemma 1 applies when $A$ is primitive and $K=\operatorname{soc}(A)$.

THEOREM 10. Let $A$ be a primitive Banach algebra with unit. If $T \in A$ and $p$ is a polynomial with $p(T) \in \operatorname{soc}(A)$, then there exists $J \in \operatorname{soc}(A)$ such that $p(T-J)=0$.

Proof. As noted above, we may assume that $A$ is a subalgebra of $\mathscr{A}(X, \Gamma)$ and that $\operatorname{soc}(A)=\mathscr{T}(X, \Gamma)$. We verify that $H_{N}$ holds relative to $\mathscr{T}(X, \Gamma)$ for $N \geq 1$. Suppose that $T^{n} \in \mathscr{T}(X, \Gamma)$ and $E=E^{2} \in A$ with $E T E=T$. By Lemma $9 \exists G \in \mathscr{T}(X, \Gamma)$ such that $(T(I-G))^{n}=0$. Then $[E T(E-E G E)]^{n}=0$. Thus $T=T(I-G) E+T G E, T G E \in \mathscr{T}(X, \Gamma)$, $E(T(I-G) E)=T(I-G) E$, and $(T(I-G) E)^{n}=0$. This verifies $H_{N}$.

Finally, since $p(T) \in \mathscr{T}(X, \Gamma)$, we have $\sigma(p(T))$ is finite, and thus $\sigma(T)$ is finite. Therefore Lemma 1 applies.

Recall that $\mathscr{T}(X)$ is the ideal of bounded operators on $X$ with finite dimensional range. The following corollary was noted by Olsen [12, Theorem 4.5] in the case where $X$ is a Hilbert space.

Corollary 11. Let $X$ be a Banach space. If $T \in \mathscr{B}(X)$ and $p$ is a polynomial with $p(T) \in \mathscr{T}(X)$, then $\exists J \in \mathscr{T}(X)$ such that $p(T-J)=0$.

Now we extend Theorem 10 to the case where $A$ is semisimple.

THEOREM 12. Let $A$ be a semisimple Banach algebra with unit. Let $K$ be an ideal of $A$ with $K \subset \operatorname{soc}(A)$. If $f \in A$ and $p$ is a polynomial with $p(f) \in K$, then $\exists h \in K$ such that $p(f-h)=0$.

Proof. The ideal $K$ is an algebraic direct sum of minimal ideals of $A$. There exists a finite set of minimal ideals of $A,\left\{M_{1}, \ldots, M_{n}\right\}$, such that 
$p(f) \in M_{1} \oplus \cdots \oplus M_{n}$. Let $I_{j}=\left\{g \in A: g M_{j}=\{0\}\right\}$. Let $\pi_{j}$ be the quotient projection, $\pi_{j}: A \rightarrow A / I_{j}$. Then $\pi_{j}(A)$ is a primitive Banach algebra with unit and $\pi_{j}\left(M_{j}\right)=\operatorname{soc}\left(\pi_{j}(A)\right)$. Also,

$$
p\left(\pi_{j}(f)\right)=\pi_{j}(p(f)) \in \pi_{j}\left(M_{j}\right) .
$$

Therefore by Theorem $10 \exists h_{j} \in M_{j}$ such that

$$
\pi_{j}\left(p\left(f-h_{j}\right)\right)=p\left(\pi_{j}\left(f-h_{j}\right)\right)=0 .
$$

Thus $p\left(f-h_{j}\right) \in I_{j}$. Let $h=\sum_{j=1}^{n} h_{j}$. Now

$$
p(f-h) \in M_{1} \oplus \cdots \oplus M_{n} .
$$

Since $p\left(f-h_{k}\right) \in I_{k}$, we have for $w_{k} \in M_{k}$,

$$
p(f-h) w_{k}=p\left(f-h_{k}\right) w_{k}=0 .
$$

It follows that

$$
p(f-h) \in\left[\bigcap_{k=1}^{n} I_{k}\right] \cap\left[M_{1} \oplus \cdots \oplus M_{n}\right]=\{0\} .
$$

We note one application of this result. Let $G$ be a compact group, and let $M(G)$ be the measure algebra on $G$ [7, p. 269]. Then $\operatorname{soc}(M(G))=$ $\mathscr{T}(G)$, the ideal of all trigonometric polynomials on $G[8, \mathrm{p} .5]$. This is verified in [3, Lemma A.6.1].

CoRollary 13. If $\mu \in M(G)$ and $p$ is a polynomial with $p(\mu) \in \mathscr{T}(G)$, then $\exists f \in \mathscr{T}(G)$ such that $p(\mu-f)=0$.

3. Applications to $C^{*}$-algebras. Throughout this section we assume that $A$ is a $C^{*}$-algebra. When $A=\mathscr{B}(H)$, then $\overline{\operatorname{soc}(A)}=\mathscr{K}(H)$. Olsen's Theorem [12, Theorem 2.4] involves algebraic elements modulo $\overline{\operatorname{soc}(A)}$ in this specific situation. We prove a result that generalizes Olsen's Theorem: If $A$ is a general $C^{*}$-algebra and $K$ is a closed ideal, $K \subset \overline{\operatorname{soc}(A)}$, then if $p$ is a polynomial with $p(T) \in K$, then $\exists J \in K$ such that $p(T-J)=0$.

First we prove a lemma.

Lemma 14. Let $K$ be an ideal of $A$. Assume that $e=e^{2} \in A, E=E^{*}=$ $E^{2} \in A$ and that

(1) $E e=e$ and $e E=e$.

Assume further that the ideal EKE in the $C^{*}$-algebra EAE has the property:

(2) if $k \in E A E$ and $k^{n} \in E K E$, then $\exists h \in E K E$ such that $(k-h)^{n}=$ 0 .

If $f=e f e$ and $f^{n} \in K$, then $\exists g \in A$ with $g=e g e, g^{n}=0$, and $\exists h \in K$ such that $f=g+h$. 
Proof.

$$
f^{n}=(f e)^{n-1} f=(f E e)^{n-1} f=(f E)^{n-1} f .
$$

Therefore $(E f E)^{n}=E f^{n} E \in E K E$. By (2) $E f E=E g E+E h E$ where $(E g E)^{n}=0$ and $h \in K$. Now

$$
f=e f=E e f=E f e=E g e+E h e .
$$

Also, $e(E g e) e=E g e$,

$$
(E g e)^{n}=(E g)^{n} e=(E g E)^{n} e=0,
$$

and $E h e \in K$.

If $A$ is a semisimple normed algebra and $E=E^{2} \in A$, then it is not difficult to prove that

$$
\operatorname{soc}(E A E)=E \operatorname{soc}(A) E .
$$

We use this fact in the proof of the theorem.

TheOREM 15. Let $A$ be a $C^{*}$-algebra with unit, and let $K$ be a closed ideal of $A$ such that $K \subset(\operatorname{soc}(A))^{-}$. If $T \in A$ and $p$ is a polynomial with $p(T) \in K$, then $\exists J \in K$ such that $p(T-J)=0$.

Proof. Since $K \subset(\operatorname{soc}(A))^{-}, K$ is a Riesz-algebra [3, p. 60]. This implies that $\sigma(p(T))$ is countable. Therefore $\sigma(T)$ is countable by the Spectral Mapping Theorem. Thus (2) of Theorem 1 is automatically satisfied for $T$. We verify that $K$ satisfies $H_{N}$ for any $N$. Using the construction given in [3, pp. 79-81], $A$ has a representation as a closed *-subalgebra of $B(H)$ with the properties:

(i) there is an index set $\Lambda$ such that $\left\{H_{\lambda}: \lambda \in \Lambda\right\} \cup\left\{H_{0}\right\}$ is a collection of mutually orthogonal closed $A$-invariant subspaces of $H$ with $H=H_{0} \oplus \sum_{\lambda \in \Lambda} H_{\lambda}$ (Hilbert space direct sum);

(ii) for all $\lambda \in \Lambda, A \mid H_{\lambda} \supset \mathscr{K}\left(H_{\lambda}\right)$.

Also, using the fact that $K$ is a Riesz (or annihilator, or dual) $C^{*}$-algebra, we have from [13, Theorem (4.10.14)]:

(iii) $R \in K$ if and only if $R_{\lambda}=R \mid H_{\lambda} \in \mathscr{K}\left(H_{\lambda}\right)$ for all $\lambda \in \Lambda$, and for each $\varepsilon>0$,

$$
\left\{\lambda \in \Lambda:\left\|R_{\lambda}\right\| \geq \varepsilon\right\} \text { is finite. }
$$

Let $V$ be the von-Neumann algebra generated by $A$ as a subalgebra of $\mathscr{B}(H)$. The characterization of $K$ in (iii) implies the key fact that

$K$ is a closed ideal in $V$.

Assume $T^{n} \in K$. By [10, Theorem 2] $\exists E=E^{*}$ a projection in $V$ such that

$$
T^{n-1} E \in K \text { and }(I-E) T \in K \text {. }
$$


We prove that $\exists J \in K$ such that $(T-J)^{n}=0$ by induction. Certainly this is true if $n=1$. Assume it holds true for $n-1$. Now let $E$ be as above.

$$
T=E T+(I-E) T \text { and }(I-E) T \in K .
$$

Therefore $\exists J_{0} \in K$ such that $T^{n-1}=(E T)^{n-1}+J_{0}$. Thus

$$
(E T E)^{n-1}=\left(E T^{n-1} E-E J_{0} E\right) \in E K E .
$$

As noted before the statement of the theorem, $\operatorname{soc}(E V E)=E \operatorname{soc}(V) E$. Therefore $E K E$ is a closed ideal in the closure of $\operatorname{soc}(E V E)$. Applying the induction hypothesis we have $\exists J_{1} \in E K E$ such that $\left(E T E-J_{1}\right)^{n-1}=0$. Set $S_{1}=E T E-J_{1}$ (note $E S_{1} E=S_{1}$ ). Let $J=J_{1}+(I-E) T \in K$, and $S=T-J=E S_{1} E+E T(I-E)$. Then

$$
\begin{aligned}
S^{n} & =\left[E S_{1} E+E T(I-E)\right]^{n} \\
& =\left(E S_{1} E\right)^{n}+\left(E S_{1} E\right)^{n-1} E T(I-E)=0 .
\end{aligned}
$$

Now suppose $T^{n} \in K$ and $e=e^{2} \in A$ with $T=e T e$. Choose $E=E^{*}$ $=E^{2} \in A$ such that $E e=e$ and $e E=E[14$, Theorem 6.1]. We have $E K E$ is a closed ideal contained in the closure of the socle of the $C^{*}$-algebra $E A E$. Thus the previous argument establishes that (2) of Lemma 14 holds. Then it follows from that lemma that $H_{N}$ is true for $K$ for all $N$.

The West Decomposition in a $C^{*}$-algebra $A$ states that if $K$ is a closed ideal, $K \subset \overline{\operatorname{soc}(A)}$, and $f$ is quasinilpotent modulo $K$, then $f=q+h$ where $q$ is quasinilpotent $\left(\left\|q^{n}\right\|^{1 / n} \rightarrow 0\right.$ as $\left.n \rightarrow \infty\right)$ and $h \in K\left[3, C^{*} .2 .5\right]$. We note that in this same situation, Theorem 15 implies that when $f^{n} \in K$, then $f=g+h$ where $g^{n}=0$ and $h \in K$. Whether this property holds when $K$ is an arbitrary closed ideal is an open question [1, Question 2.7]. Akemann and Pedersen prove that this is so for $n=2$ [1, Prop. 2.8]. We use their result and Lemma 1 to prove our next theorem. If the question of Akemann and Pedersen [1, Question 2.7] has an affirmative answer, then the next result would be true with no restriction on the integers $n_{j}$.

Theorem 16. Let $A$ be $a C^{*}$-algebra and $K$ be a closed ideal of $A$. let

$$
p(z)=\prod_{j=1}^{m}\left(z-\lambda_{j}\right)^{n_{j}}
$$

where $n_{j}=1$ or 2 for all $j$. If $f \in A$ has properties (1) and (2) listed in Lemma 1 , then $f=g+h$ where $p(g)=0$ and $h \in K$.

Proof. It is enough to verify $\mathrm{H}_{2}$ holds for $K$. Assume $f \in A, e^{2}=e \in$ $A, f=e f e$, and $f^{2} \in K$. By [14, Theorem 6.1] there exists $E=E^{*}=E^{2} \in$ $A$ such that $E e=e$ and $e E=E$. Also, by the Akemann-Pederson result 
[1, Prop. 2.8] property (2) of Lemma 14 is satisfied with $n=2$. Thus by that lemma, $\exists g \in A$ with $g=e g e, g^{2}=0$, and $\exists h \in K$ such that $f=$ $g+h$.

\section{REFERENCES}

[1] C. Akemann and G. Pedersen, Ideal perturbations of elements in $C^{*}$-algebras, Math. Scand., 41 (1977), 117-139.

[2] B. Barnes, When is a representation of a Banach *-algebra Naimark-related to a *-representation?, Pacific J. Math., 72 (1977), 5-25.

[3] B. Barnes, G. Murphy, M. Smyth, and T. West, Riesz and Fredholm Theory in Banach Algebras, Research Notes in Mathematics 67, Pitman, London, 1982.

[4] F. Bonsall and J. Duncan, Complete Normed Algebras, Springer-Verlag, Berlin, 1973.

[5] J. Calkin, Two-sided ideals and congruences in the ring of bounded operators in Hilbert spaces, Ann. of Math., (2) 42 (1941), 839-873.

[6] S. Caradus, W. Pfaffenberger, and B. Yood, Calkin Algebras and Algebras of Operators on Banach Spaces, Lecture Notes in Mathematics, Vol. 9, Marcel Dekker, Inc., N. Y., 1974.

[7] E. Hewitt and K. Ross, Abstract Harmonic Analysis, Vol. I, Springer-Verlag, Berlin, 1963.

[8] _ Abstract Harmonic Analysis, Vol. II, Springer-Verlag, Berlin, 1970.

[9] E. Hille, On roots and logarithms of elements of a complex Banach algebra, Math. Ann., 136 (1958), 46-57.

[10] Y. Kato, A proof of Olsen's Theorem, Math. Japonicae, 21 (1976), 127-128.

[11] R. Miers, Polynomial ideal $C^{*}$-algebras, Amer. J. Math., 98 (1976), 165-170.

[12] C. Olsen, A structure theorem for polynomial compact operators, Amer. J. Math., 93 (1971), 686-698.

[13] C. Rickart, General Theory of Banach Algebras, Van Nostrand, N. Y., 1960.

[14] R. Smyth, Riesz theory in Banach algebras, Math. Zeit., 145 (1975), 145-155.

Received May 10, 1983 and in revised form December 24, 1984.

UNIVERSITY OF OREGON

EUGENE, OR 97403 



\section{PACIFIC JOURNAL OF MATHEMATICS EDITORS}

DONALD BABBITT (Managing Editor)
University of California
Los Angeles, CA 90024
CHARLES R. DEPrIMA
California Institute of Technology
Pasadena, CA 91125
R. FINN
Stanford University
Stanford, CA 94305

\author{
HERMANN FLASCHKa \\ University of Arizona \\ Tucson, AZ 85721 \\ RAMESH A. GANGOLLI \\ University of Washington \\ Seattle, WA 98195 \\ ROBION KIRBY \\ University of California \\ Berkeley, CA 94720 \\ C. C. Moore \\ University of California \\ Berkeley, CA 94720
}

Hugo Rossi

University of Utah

Salt Lake City, UT 84112

H. SAMELSON
Stanford University
Stanford, CA 94305

HAROLD STARK

University of California, San Diego

La Jolla, CA 92093

\section{ASSOCIATE EDITORS}
R. ARens
E. F. BECKENBACH (1906-1982)
B. H. NeUmanN
F. WOLF
K. YOSHIDA

\begin{tabular}{ll}
\multicolumn{2}{c}{ SUPPORTING INSTITUTIONS } \\
UNIVERSITY OF ARIZONA & UNIVERSITY OF OREGON \\
UNIVERSITY OF BRITISH COLUMBIA & UNIVERSITY OF SOUTHERN CALIFORNIA \\
CALIFORNIA INSTITUTE OF TECHNOLOGY & STANFORD UNIVERSITY \\
UNIVERSITY OF CALIFORNIA & UNIVERSITY OF HAWAII \\
MONTANA STATE UNIVERSITY & UNIVERSITY OF TOKYO \\
UNIVERSITY OF NEVADA, RENO & UNIVERSITY OF UTAH \\
NEW MEXICO STATE UNIVERSITY & WASHINGTON STATE UNIVERSITY \\
OREGON STATE UNIVERSITY & UNIVERSITY OF WASHINGTON
\end{tabular}

The Supporting Institutions listed above contribute to the cost of publication of this Journal, but they are not owners or publishers and have no responsibility for its content or policies.

\footnotetext{
Mathematical papers intended for publication in the Pacıfic Journal of Mathematıcs should be in typed form or offset-reproduced (not dittoed), double spaced with large margins. Please do not use built up fractions in the text of the manuscript. However, you may use them in the displayed equations. Underline Greek letters in red, German in green, and script in blue. The first paragraph must be capable of being used separately as a synopsis of the entire paper. In particular it should contain no bibliographic references. Please propose a heading for the odd numbered pages of less than 35 characters. Manuscripts, in triplicate, may be sent to any one of the editors. Please classify according to the scheme of Math. Rev ews, Index to Vol. 39. Supply name and address of author to whom proofs should be sent. All other communications should be addressed to the managing editor, or Elaine Barth, University of California, Los Angeles, California 90024.

There are page-charges associated with articles appearing in the Pacific Journal of Mathematics. These charges are expected to be paid by the author's University, Government Agency or Company. If the author or authors do not have access to such Institutional support these charges are waived. Single authors will receive 50 free reprints; joint authors will receive a total of 100 free reprints. Additional copies may be obtained at cost in multiples of 50 .
}

The Pacific Journal of Mathematics is issued monthly as of January 1966. Regular subscription rate: $\$ 190.00$ a year (5 Vols., 10 issues). Special rate: $\$ 66.00$ a year to individual members of supporting institutions.

Subscriptions, orders for numbers issued in the last three calendar years, and changes of address should be sent to Pacific Journal of Mathematics, P.O. Box 969, Carmel Valley, CA 93924, U.S.A. Old back numbers obtainable from Kraus Periodicals Co., Route 100, Millwood, NY 10546.

The Pacific Journal of Mathematics at P.O. Box 969, Carmel Valley, CA 93924 (ISSN 0030-8730) publishes 5 volumes per year. Application to mail at Second-class postage rates is pending at Carmel Valley, California, and additional mailing offices. Postmaster: Send address changes to Pacific Journal of Mathematics, P.O. Box 969, Carmel Valley, CA 93924.

PUBLISHED BY PACIFIC JOURNAL OF MATHEMATICS, A NON-PROFIT CORPORATION Copyright $\odot 1985$ by Pacific Journal of Mathematics 


\section{Pacific Journal of Mathematics}

\section{Vol. 117, No. 2 \\ February, 1985}

Robert Walter Bagley, Ta-Sun Wu and J. S. Yang, On a class of topological groups more general than SIN groups ............... 209

Bruce Alan Barnes, Algebraic elements of a Banach algebra modulo an ideal ...............................................219

Howard D. Fegan and Peter Gilkey, Invariants of the heat equation .......233

Erica Flapan, Necessary and sufficient conditions for certain homology

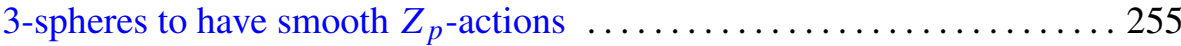

Philip R. Heath, Product formulae for Nielsen numbers of fibre maps . . . . 267

Derbiau Frank Hsu and A. Donald Keedwell, Generalized complete mappings, neofields, sequenceable groups and block designs. II ...... 291

Taqdir Husain, Orthogonal primitive idempotents and Banach algebras

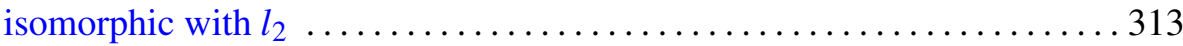

Gary M. Lieberman, Regularized distance and its applications . . . . . . . 329

William W. Menasco, Determining incompressibility of surfaces in alternating knot and link complements ............................ 353

Benjamin Muckenhoupt, Weighted reverse weak type inequalities for the Hardy-Littlewood maximal function $\ldots \ldots \ldots \ldots \ldots \ldots \ldots \ldots \ldots \ldots \ldots \ldots$

John Dacey O'Neill, Direct summands of direct products of slender

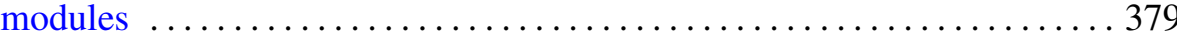

Andreas Rapp, Elimination of Malitz quantifiers in stable theories 387

Francisco José Ruiz, A unified approach to Carleson measures and $A_{p}$ weights

Hanamatagouda Pandappa Sankappanavar, Heyting algebras with dual

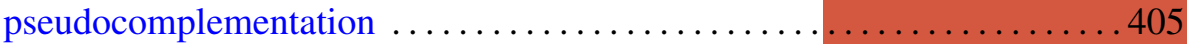

Annette Sinclair, Strong Carleman and strong uniform approximation . . . . 417

Masaaki Suzuki, The generalized Schwarz lemma for the Bergman metric

Brian Thorpe and Ludwig Tomm, Universal approximation by regular

weighted means 